\title{
Chemically Modified mocRNAs for Highly Efficient Protein Expression in Mammalian Cells
}

\author{
Abhishek Aditham ${ }^{1,2,7}$, Hailing Shi ${ }^{1,3,7}$, Jianting Guo ${ }^{1,3}$, Hu Zeng1, Yiming Zhou ${ }^{1}$, Sarah Dunn \\ Wade $^{1,4,5}$, Jiahao Huang ${ }^{1}$, Jia Liu ${ }^{6}$, Xiao Wang ${ }^{1,3,4 *}$
}

\author{
Affiliations: \\ ${ }^{1}$ Broad Institute of MIT and Harvard, Cambridge, MA 02142, USA. \\ ${ }^{2}$ Department of Biological Engineering, Massachusetts Institute of Technology, Cambridge, MA 02142, \\ USA. \\ ${ }^{3}$ Department of Chemistry, Massachusetts Institute of Technology, Cambridge, MA 02139, USA. \\ ${ }^{4}$ Stanley Center for Psychiatric Research, Broad Institute of MIT and Harvard, Cambridge, MA 02142, \\ USA \\ ${ }^{5}$ Department of Neuroscience, University of California San Francisco, San Francisco, CA 94158, USA \\ ${ }^{6}$ John A. Paulson School of Engineering and Applied Sciences, Harvard University, Cambridge, MA \\ 02134, USA \\ ${ }^{7}$ These authors contributed equally \\ *e-mail: xwangx@mit.edu
}

\section{ORCID:}

Abhishek Aditham (0000-0003-3203-4574), Hailing Shi (0000-0001-7355-378X), Jianting Guo (00000002-5691-5707), Hu Zeng (0000-0003-4773-205X), Yiming Zhou (0000-0002-8365-0396), Sarah Dunn Wade (0000-0002-5746-5111), Jiahao Huang (0000-0002-6349-9799), Jia Liu (0000-0003-2217-6982), Xiao Wang (0000-0002-3090-9894) 


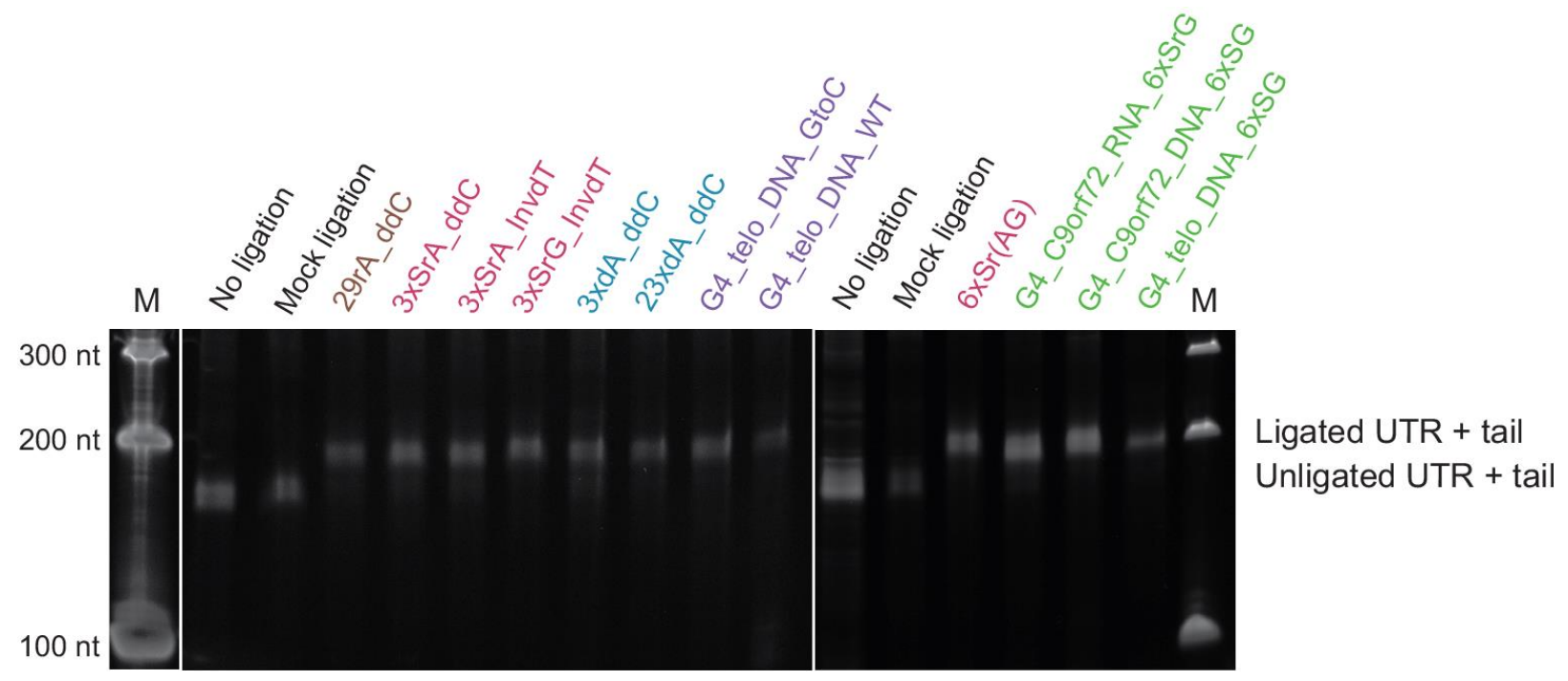

Supplementary Figure 1 | Representative RNase H characterization of mocRNAs ligated to a panel

of synthetic oligonucleotides. Representative RNase H assays showing mocRNA vectors prepared by the ligation of IVT GFP-60A mRNAs and synthetic oligos. DNA probe targets the 3' UTR of mRNA such that the 5 ' end of the probe is $106 \mathrm{nt}$ upstream of the poly(A) tail. This generates a 5' mRNA fragment (824 nt) and a 3' mRNA fragment (166 nt including $60 \mathrm{nt}$ poly(A), Lanes $1 \&$ 2). The 3' cleavage product displays a band shift on a denaturing gel upon ligation. M, Marker which is Century-Plus RNA Markers. Ligated and unligated tails are labeled accordingly. 
a Single-cell fluorescent protein analysis

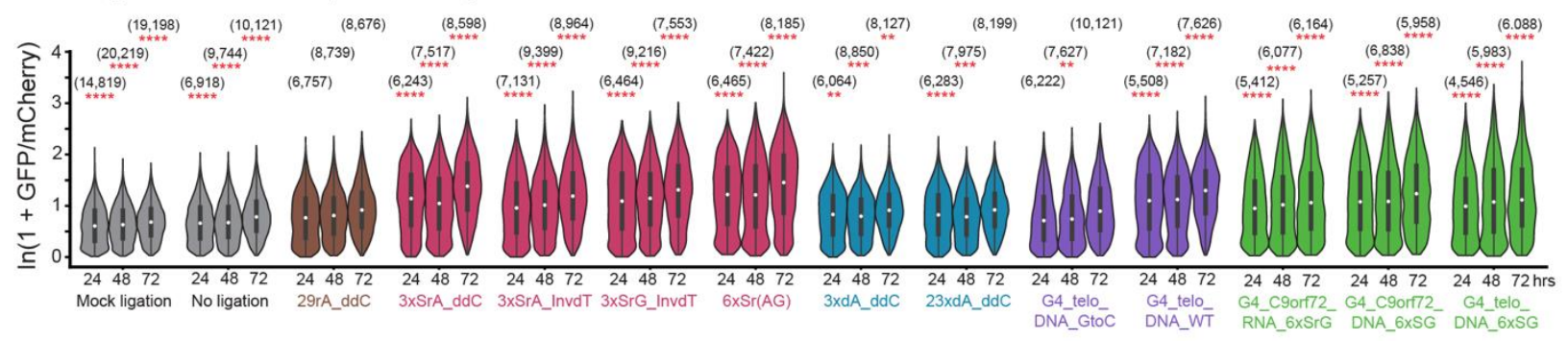

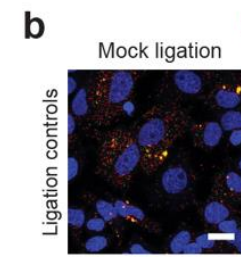

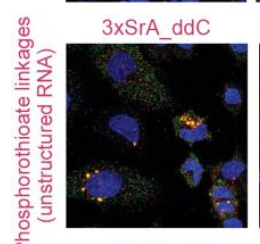

3xdA ddC

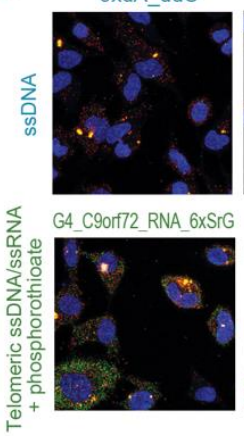

mCherry mRNA / GFP mRNA / DAPI

No ligation mCherry mRNA only

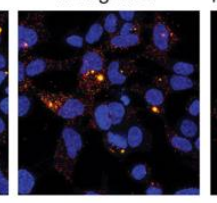

3xSrA_InvdT

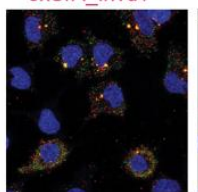

23xdA ddC

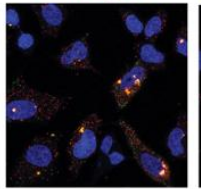

G4_C9or72_DNA_6xS

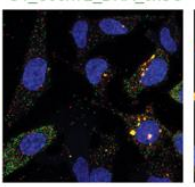

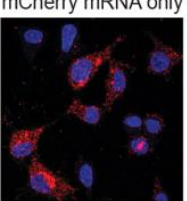

3xSrG_InvdT

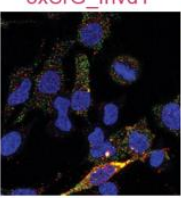

G4 telo DNA Gtoc

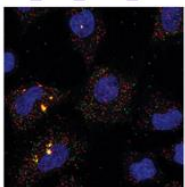

G4 telo DNA 6xSG

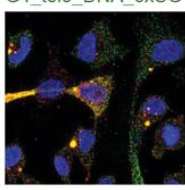

C Single-cell mRNA quantification

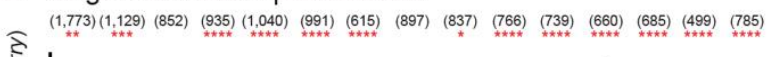

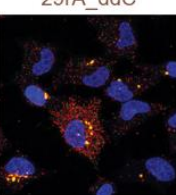

$6 x \operatorname{Sr}(A G)$

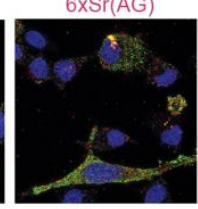

G4 telo DNA WT

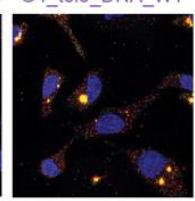

d

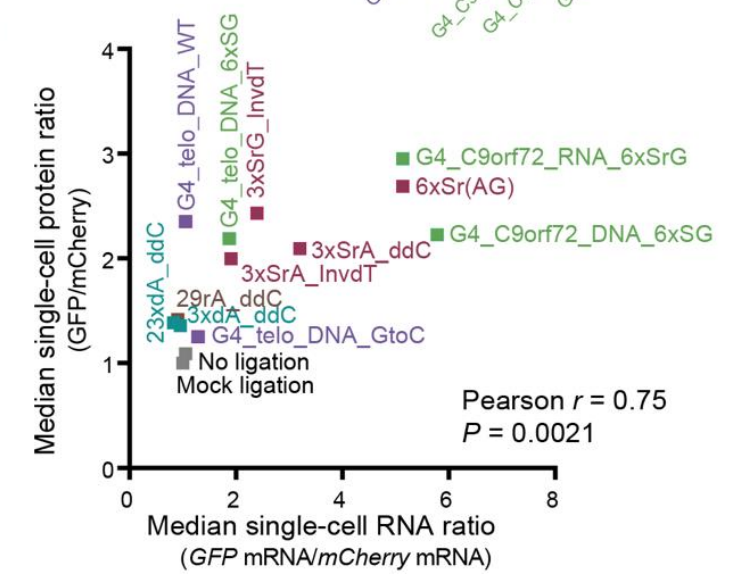

Supplementary Figure 2 | Single-cell quantification of protein production and RNA abundance from

mocRNA vectors in HeLa cells. (a) Violin plots of single-cell quantification of GFP and mCherry fluorescence ratios $(\ln [1+$ ratio] $)$ in HeLa cells 24 hours, 48 hours, and 72 hours after transfected with indicated mRNA vectors. Violin plot elements, lines, lower/upper adjacent values; bars, interquartile ranges; white dot, median. $n$ indicated in parentheses. $P$ values are calculated by Welch's $t$ test (unpaired, two-tailed), with comparisons to the sample 29rA_ddC as a control. ** $P<0.01$, *** $P<0.001$, ****P< 0.0001. (b) Representative image stack maximum projection of STARmap characterization of GFP and $m$ Cherry RNA in HeLa cells 48 hours after lipofectamine-mediated transfection. GFP and $m$ Cherry mRNA species trapped in lipofectamine-mediated vesicles appeared overlapped and formed large, merged foci. 
mRNA species released from the vesicle appeared as individual dots in the cytosol, each representing a single mRNA molecule. Scale bar, $20 \mu \mathrm{m}$. (c) Single-cell analysis of GFP/mCherry mRNA copy numbers (amplicons) quantified by STARmap. Violin plot elements: lines, lower/upper adjacent values; bars, interquartile ranges; white dot, median. Number of cells in parentheses. Gray dash line, median of the sample 29rA_ddC. $P$ values are calculated by Welch's t test (unpaired, two-tailed), with comparisons to the sample 29rA_ddC as a control. $* P<0.05$, *** $P<0.01$, *** $P<0.001$, **** $P<0.0001$. (d) Correlation of the medians of single-cell GFP/mCherry RNA ratios and single-cell GFP/mCherry fluorescence ratios 48 hours after transfection. 
a

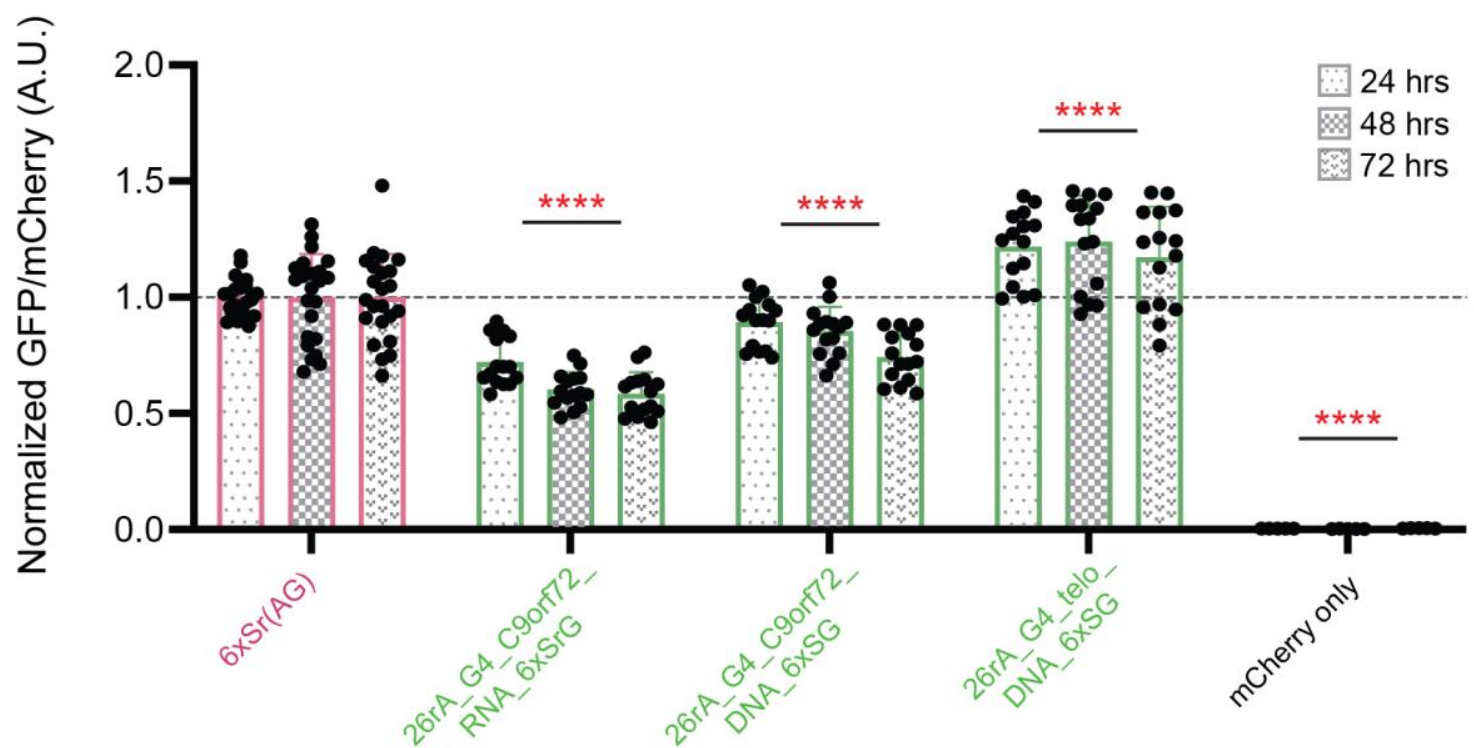

b In vitro translation of mocRNA

c Renilla luciferase internal control (Fig. 3b)
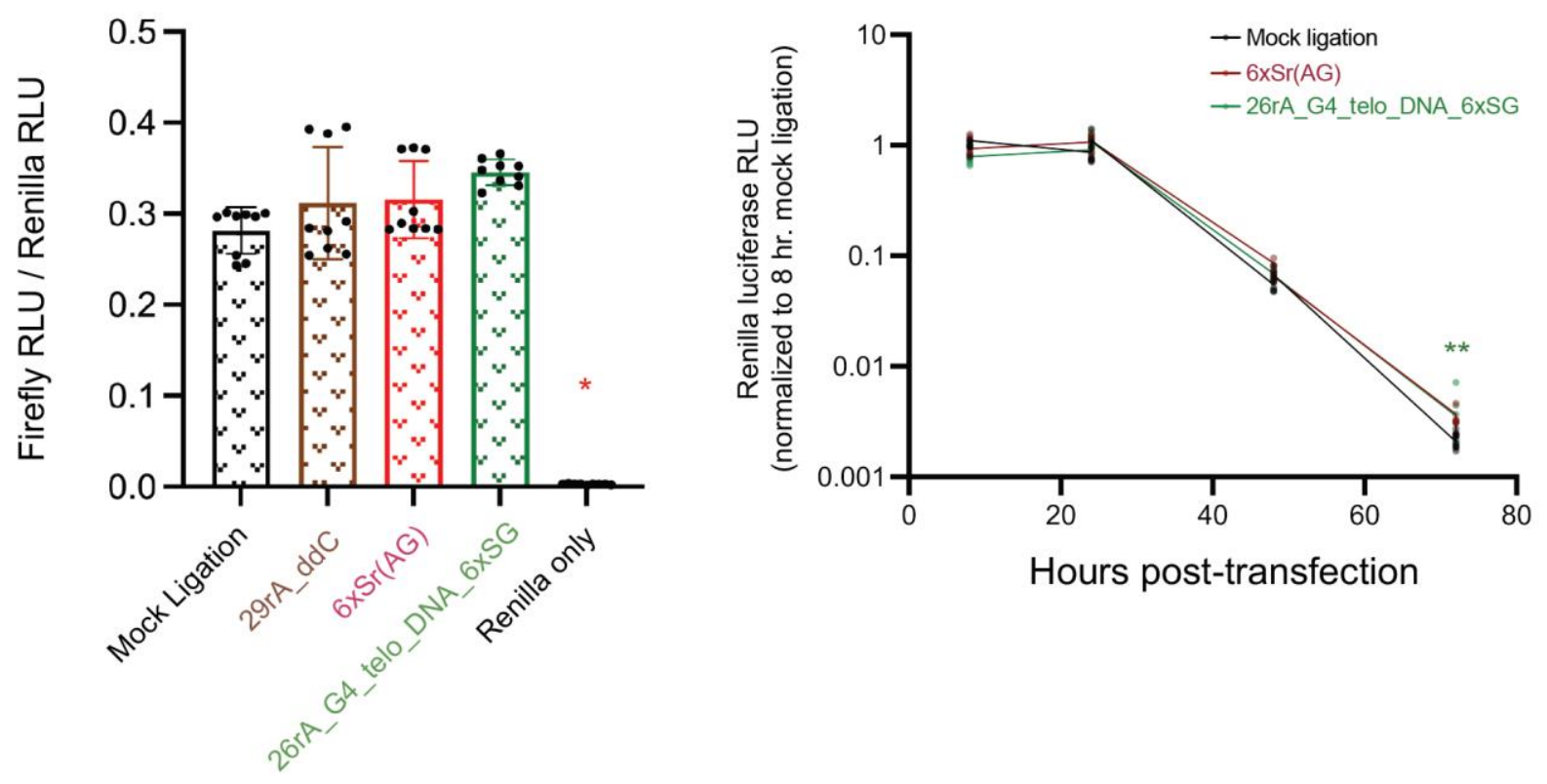

Supplementary Figure 3 | Kinetic characterization of length-adjusted phosphorothioate and telomeric (G4) oligos. (a) GFP-60A mocRNAs ligated to length-adjusted PS+G4 oligos (26rA_G4_C9orf72_RNA_6xSrG, 26rA_G4_C9orf72_DNA_6xSG, and 26rA_G4_telo_DNA_6xSG). Fluorescence time-course measurements were performed following transfection of GFP mocRNAs into HeLa cells, along with an mCherry mRNA internal control. Resulting GFP/mCherry fluorescence values for each sample were further normalized to the average value for $6 \mathrm{x} \operatorname{Sr}(\mathrm{AG})$ at each time point. Statistical 
testing was performed using ordinary two-way ANOVA (Dunnett's multiple comparisons test, comparison of means across timepoints), with comparisons performed to $6 \mathrm{xSr}(\mathrm{AG})$. $* * * * P<0.0001$. (b) In vitro translation of Firefly-PEST mocRNA constructs. Rabbit reticulocyte lysates were used as in vitro translation systems for Firefly-PEST mocRNA constructs, along with an unmodified internal Renilla luciferase control. Firefly RLU / Renilla RLU were measured from each reaction to compare possible modes of translational enhancement afforded by different mocRNAs. Statistical testing was performed using one-way ANOVA (nonparametric, Kruskal-Wallis, Dunn's multiple comparisons test), with comparisons made to the "mock ligation" sample. $* P<0.05$. (c) Kinetic characterization of Firefly-degron encoding mocRNA constructs. Renilla (internal control) RLU normalized to mock ligation value at 8 hours post-transfection. Corresponding mocRNA values at each timepoint were tested for significance using a one-way ANOVA (Kruskal-Wallis test, Dunn's multiple comparisons test), compared to mock ligation. The internal control signal appeared to be consistent between different samples. 


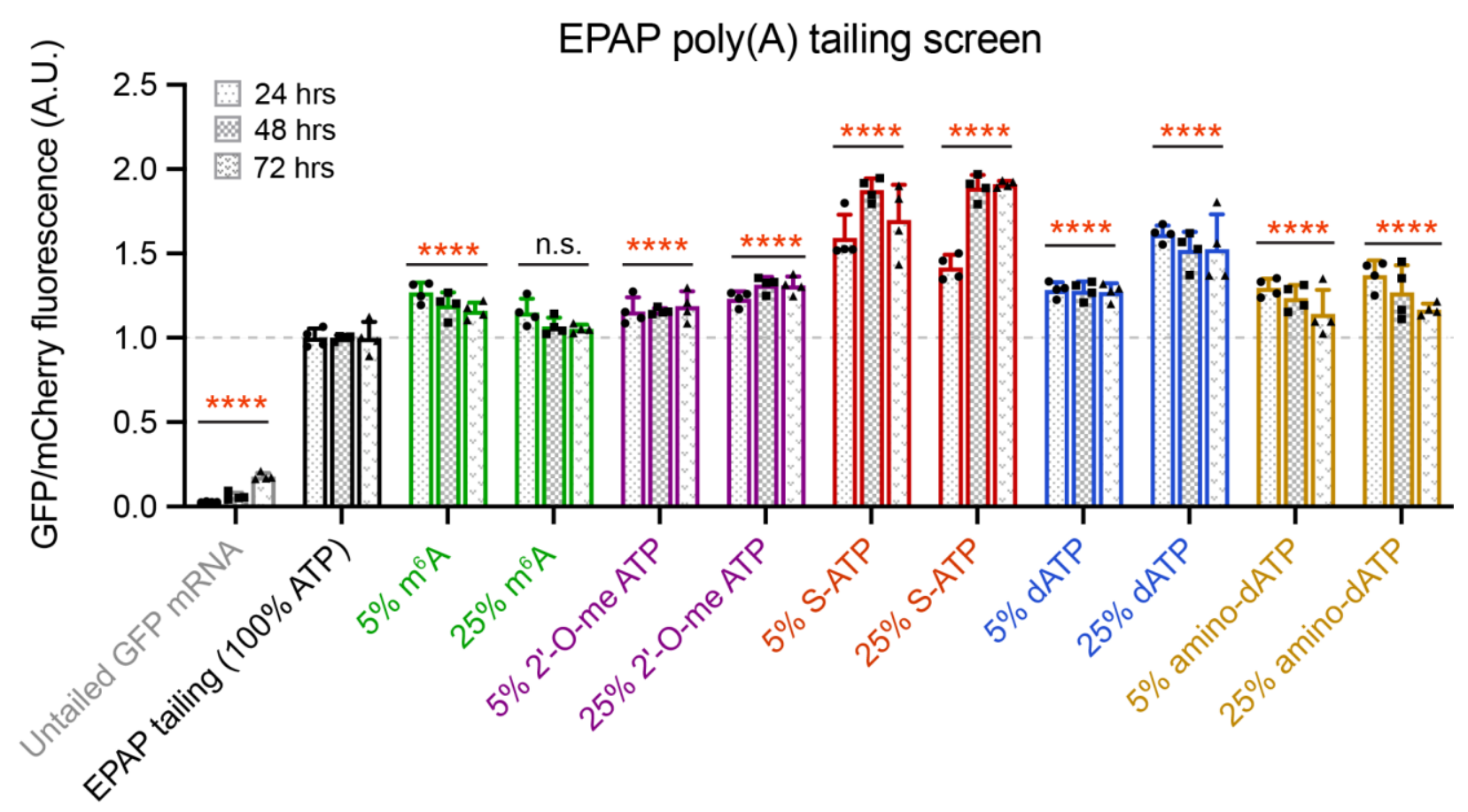

\section{Supplementary Figure 4 | Protein expression of mRNAs containing E-PAP-modified poly(A) tails.}

GFP mRNAs were subjected to poly(A) tailing by E. coli poly(A) polymerase (E-PAP), with varying amounts of chemically modified ATP derivatives spiked in. Tail-modified GFP mRNAs were transfected into HeLa cells, along with tail-unmodified mCherry transfection control (E-PAP tailed, 100\% ATP). Bars represent GFP/mCherry fluorescence normalized by the average of the $100 \%$ ATP, E-PAP tailed GFP mRNA sample at each corresponding time point. The percentages indicate the relative molar ratio used between modified and unmodified ATP in each reaction. Chemically modified GFP mRNAs were cotransfected with unmodified mCherry mRNA, and the resulting GFP/mCherry fluorescence ratios were measured at 24, 48, and 72 hours post transfection in HeLa cell culture. ATP: adenosine 5' triphosphate; m6 ATP: $N^{6}$-methyladenosine 5' triphosphate; 2'-O-me ATP: 2' O-methyladenosine-5'-triphosphate; SATP: adenosine-5'-O-(1-thiotriphosphate); dATP: 2'-deoxyadenosine 5'-triphosphate; amino-dATP: 2'amino-2'-deoxyadenosine-5'-triphosphate. mean \pm s.d. $n=4$. Gray dash line: $y=1 . P$ values are calculated by ordinary two-way ANOVA (Dunnett's multiple comparisons test, comparison of means across timepoints), with comparisons performed to E-PAP tailing (100\% ATP). $* * * * P<0.0001$. 

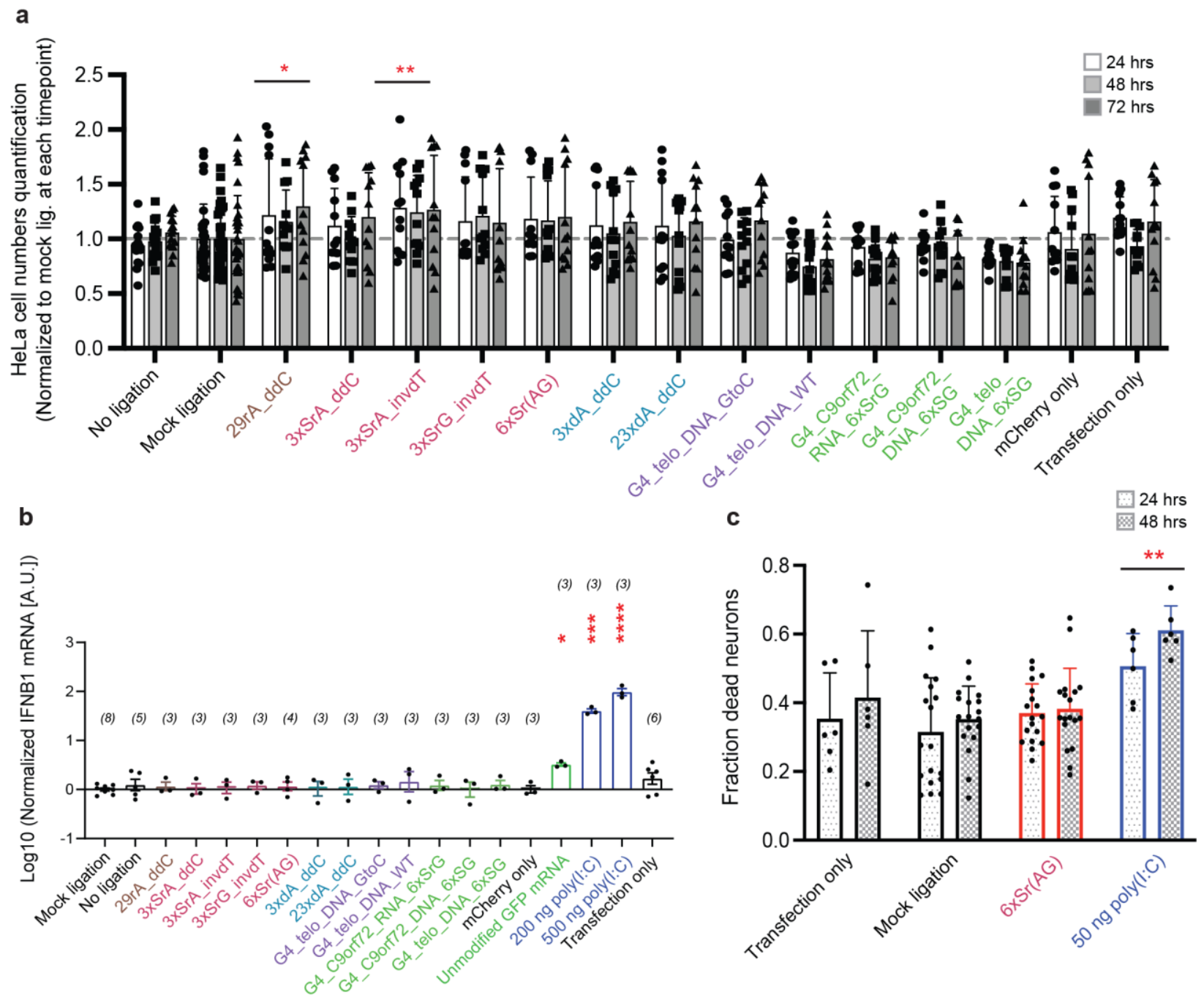

Supplementary Figure 5 | mocRNA toxicity profiling in HeLa and primary rat cortical neurons. (a)

Quantification of HeLa cell numbers from confocal microscopy images (Fig. 2). Hoechst-stained nuclei were segmented in CellProfiler, and cell numbers in each field of view (FOV) were calculated for each mocRNA condition and time point. Cell numbers were normalized to average cell number for the mock ligation condition at every time point. Comparisons were performed to the "no ligation" sample using an ordinary two-way ANOVA (Dunnett's multiple comparisons test, comparison of means across timepoints). $* P<0.05, * * P<0.01$. (b) RT-qPCR quantification of innate immune response in transfected HeLa cells. RT-qPCR of IFNB1 mRNA in samples transfected with each GFP-60A ligation construct, normalized to human $A C T B$ mRNA and normalized again to the mock ligation sample. Values were further $\log 10$ transformed prior to significance testing and graphing. Each condition consists of at least 3 biological 
replicates, with 3 technical replicates per biological sample. Averages of 3 technical replicates (for each biological condition) are shown as individual points, such that each data point corresponds to a specific biological replicate (mean + s.e.m of biological replicates). Unmodified GFP mRNA refers to IVT hMGFP mRNA (E-PAP poly(A) tailed) without $N^{1}$-methylpseudouridine substitution (i.e., contains $100 \%$ uridine). Log10-normalized samples were analyzed for significance using Welch's $t$ test (unpaired, two-tailed, parametric). Samples were referenced to 29rA_ddC mocRNA for pairwise comparisons. Number of biological replicates used for each condition (n) indicated in parentheses above the corresponding sample. $* P<0.05$, $* * P<0.01, * * * P<0.001, * * * * P<0.0001$. (c) Fraction of dead rat cortical neurons determined from mocRNA transfections. Primary rat cortical neuron cultures were transfected with 250 ng GFP-60A mocRNA with a $250 \mathrm{ng}$ mCherry mRNA internal control. Cells were then imaged at 24- or 48-hours posttransfection, using Hoechst to stain live and dead nuclei, and NucRed Dead (647) to stain dead nuclei. The relative numbers of dead to total nuclei were calculated to provide percentage dead cells in each transfection condition. Poly(I:C) at $50 \mathrm{ng}$ was used as a positive control for toxicity. Comparisons were performed using ordinary two-way ANOVA (Dunnett's multiple comparisons test, comparison of means across timepoints), with comparisons to the transfection only sample. $* * P<0.01$. 
3 Supplementary Table 1 | Oligonucleotides used for cloning, RT-qPCR, RNase H assays, and STARmap

4 characterization

\begin{tabular}{|c|c|}
\hline Primer name & Sequence (5' to 3') \\
\hline \multicolumn{2}{|l|}{ Cloning } \\
\hline Esp3i_insert_F & AGAGACGTTCGCGGCCGCGGCGCC \\
\hline Esp3i_insert_R & TTAAAAAACCTCCCACACCTCCCCCTGAACCTGAAAC \\
\hline 60A_insert_F & $\begin{array}{l}\text { AAAAAAAAAAAAAAAAAAAAAAAAAAAAAAAAAAAAAGAGACGT } \\
\text { TCGCGGCCGCGGCGCC }\end{array}$ \\
\hline 60A_insert_R & $\begin{array}{l}\text { TTTTTTTTTTTTTTTTTTTTTTTTTTTTTTTCСТCССАСАССТСССССТG } \\
\text { AACCTGAAAC }\end{array}$ \\
\hline \multicolumn{2}{|l|}{ RNase $\mathrm{H}$ assay } \\
\hline RNaseH_probe_GFP & GCATCACAAATTTCACAAATAAAGCATTTTTTTCAC \\
\hline \multicolumn{2}{|l|}{ RT-qPCR quantification } \\
\hline hMGFP_qPCR_F & TGACATTCTCACCACCGTGT \\
\hline hMGFP_qPCR_R & AGTCGTCCACACCCTTCATC \\
\hline mCherry_qPCR_F & TTCTTGGCCATGTAGGTGGTC \\
\hline mCherry_qPCR_R & AGGACGGCGAGTTCATCTAC \\
\hline hActb_qPCR_F & CACCATTGGCAATGAGCGGTTC \\
\hline hActb_qPCR_R & AGGTCTTTGCGGATGTCCACGT \\
\hline Origene_IFNB1_qPCR_F & CTTGGATTCCTACAAAGAAGCAGC \\
\hline Origene_IFNB1_qPCR_R & TCCTCCTTCTGGAACTGCTGCA \\
\hline \multicolumn{2}{|l|}{ STARmap: SNAIL probes } \\
\hline mCherry-01 & $\begin{array}{l}\text { /5Phos/ACATTATTGGTGCCGCGCAGCTTCACCTAATTATTACTGAGGC } \\
\text { ATACACTAAAGATA }\end{array}$ \\
\hline mCherry-02 & $\begin{array}{l}\text { /5Phos/ACATTACTTCTTGGCCTTGTAGGTGGTAATTATTACTGAGGCA } \\
\text { TACACTAAAGATA }\end{array}$ \\
\hline
\end{tabular}




\begin{tabular}{|l|l|}
\hline mCherry-03 & $\begin{array}{l}\text { /5Phos/ACATTACACGGTCACCACGCCGCCGTAATTATTACTGAGGCA } \\
\text { TACACTAAAGATA }\end{array}$ \\
\hline mCherry-11 & ACGGGGCCGTCGGAGGGGAATAATGTTATCTT \\
\hline mCherry-12 & GGCGCCGGGCAGCTGCACGGTAATGTTATCTT \\
\hline mCherry-13 & GTCCTGCAGGGAGGAGTCCTGGTAATGTTATCTT \\
\hline hMGFP-01 & $\begin{array}{l}\text { /5Phos/ACATTAAGTCGCAGCGGTAGTGGCCAATTATTACTGAAATCG } \\
\text { TAGACTAAGATA }\end{array}$ \\
\hline hMGFP-02 & $\begin{array}{l}\text { /5Phos/ACATTACATTAGCAGGGAAGTTGACCCCGTAATTATTACTGA } \\
\text { AATCGTAGACTAAGATA }\end{array}$ \\
\hline hMGFP-03 & $\begin{array}{l}\text { /5Phos/ACATTAGCTTCGGCGTGCTCGTACAGCTAATTATTACTGAAAT } \\
\text { CGTAGACTAAGATA }\end{array}$ \\
\hline hMGFP-11 & CCTCCCTCCAAGAGCAGTGCCATTAATGTTATCTT \\
\hline hMGFP-12 & TGCGCTGCATCACCGGGCTAATGTTATCTT \\
\hline hMGFP-13 & CCTGGCGGGGTAGTCCGCTGTGTAATGTTATCTT \\
\hline STARmap: fluorescent detection probes \\
\hline mCherry_detect_Alexa647 & /5Alexa647N/CATACACTAAAGATAACAT \\
\hline hMGFP_detect_Alexa546 & /5Alex546N/TCGTAGACTAAGATAACAT \\
\hline
\end{tabular}


6 Supplementary Table 2 | Statistics for GFP/mCherry fluorescence ratio data, from Fig. 2a (normalized to "mock ligation" samples)

\begin{tabular}{|c|c|c|c|c|c|c|c|c|c|c|c|c|c|c|}
\hline $\begin{array}{l}24 \quad h r \\
\text { statistics }\end{array}$ & $\begin{array}{l}\text { Mock } \\
\text { ligation }\end{array}$ & $\begin{array}{l}\text { No } \\
\text { ligati } \\
\text { on }\end{array}$ & $\begin{array}{l}\text { 29rA } \\
\text { dddC }\end{array}$ & $\begin{array}{l}\text { 3xSrA } \\
\text { dddC }\end{array}$ & $\begin{array}{l}\text { 3xSrA } \\
\text { InvdT }\end{array}$ & $\begin{array}{l}\text { 3xSrG_ } \\
\text { InvdT }\end{array}$ & $\begin{array}{l}6 \times S r( \\
\text { AG) }\end{array}$ & $\begin{array}{l}3 x d A \\
\text { ddC }\end{array}$ & $\begin{array}{l}23 x d A \\
\text { ddC }\end{array}$ & $\begin{array}{l}\text { G4_telo_D } \\
\text { NA_GtoC }\end{array}$ & $\begin{array}{l}\text { G4_telo_ } \\
\text { DNA_WT }\end{array}$ & $\begin{array}{l}\text { G4_C9orf72_- } \\
\text { RNA_6xSrG }\end{array}$ & $\begin{array}{l}\text { G4_C9orf72 } \\
\text { DNA_6xSG }\end{array}$ & $\begin{array}{l}\text { G4_telo_D } \\
\text { NA_6xSG }\end{array}$ \\
\hline Mean & 1 & 1.05 & 1.59 & 2.73 & 2.31 & 2.78 & 2.90 & 1.60 & 1.66 & 1.76 & 2.71 & 2.46 & 3.02 & 2.47 \\
\hline s.e.m & 0.006 & 0.07 & 0.13 & 0.07 & 0.16 & 0.127 & 0.074 & 0.091 & 0.088 & 0.20 & 0.078 & 0.201 & 0.23 & 0.30 \\
\hline $\begin{array}{l}48 \quad h r \\
\text { statistics }\end{array}$ & \begin{tabular}{|l|} 
Mock \\
ligation
\end{tabular} & $\begin{array}{l}\text { No } \\
\text { ligati } \\
\text { on }\end{array}$ & $\begin{array}{l}\text { 29rA } \\
\text { dddC }\end{array}$ & $\begin{array}{l}\text { 3xSrA } \\
\text { dddC }\end{array}$ & $\begin{array}{l}\text { 3xSrA } \\
\text { InvdT }\end{array}$ & $\begin{array}{l}\text { 3xSrG } \\
\text { InvdT }\end{array}$ & $\begin{array}{l}\text { 6xSr } \\
\text { AG) }\end{array}$ & $\begin{array}{l}3 x d A \\
\text { dddC }\end{array}$ & $\begin{array}{l}\text { 23xdA } \\
\text { _ddC }\end{array}$ & $\begin{array}{l}\text { G4_telo_D } \\
\text { NA_GtoC }\end{array}$ & $\begin{array}{l}\text { G4_telo_ } \\
\text { DNA_WT }\end{array}$ & $\begin{array}{l}\text { G4_C9orf72_- } \\
\text { RNA_6xSrG }\end{array}$ & $\begin{array}{l}\text { G4_C9orf72 } \\
\text { DNA_6xSG }\end{array}$ & $\begin{array}{l}\text { G4_telo_D } \\
\text { NA_6xSG }\end{array}$ \\
\hline Mean & 1 & 1.05 & 1.54 & 2.63 & 2.43 & 3.07 & 3.39 & 1.45 & 1.49 & 1.51 & 2.63 & 2.95 & 3.41 & 3.37 \\
\hline s.d. & 0.052 & 0.36 & 0.48 & 0.41 & 0.71 & 0.55 & 0.45 & 0.28 & 0.28 & 0.66 & 0.31 & 1.02 & 1.03 & 1.25 \\
\hline s.e.m. & 0.0092 & 0.081 & 0.14 & 0.12 & 0.21 & 0.16 & 0.13 & 0.081 & 0.080 & 0.19 & 0.089 & 0.29 & 0.30 & 0.36 \\
\hline $\begin{array}{l}72 \quad h r \\
\text { statistics }\end{array}$ & $\begin{array}{l}\text { Mock } \\
\text { ligation }\end{array}$ & $\begin{array}{l}\text { No } \\
\text { ligati } \\
\text { on } \\
\end{array}$ & $\begin{array}{l}\text { 29rA } \\
\text { dddC }\end{array}$ & $\begin{array}{l}\text { 3xSrA } \\
\text {-ddC }\end{array}$ & $\begin{array}{l}\text { 3xSrA } \\
\text { InvdT }\end{array}$ & $\begin{array}{l}\text { 3xSrG } \\
\text { InvdT }\end{array}$ & $\begin{array}{l}\text { 6xSr } \\
\text { AG) }\end{array}$ & $\begin{array}{l}3 x d A \\
\text { ddC }\end{array}$ & $\begin{array}{l}23 x d A \\
\text { _ddC }\end{array}$ & $\begin{array}{l}\text { G4_telo_D } \\
\text { NA_GtoC }\end{array}$ & $\begin{array}{l}\text { G4_telo_ } \\
\text { DNA_WT }\end{array}$ & $\begin{array}{l}\text { G4_C9orf72_- } \\
\text { RNA_6xSrG }\end{array}$ & $\begin{array}{l}\text { G4_C9orf72_ } \\
\text { DNA_6xSG }\end{array}$ & $\begin{array}{l}\text { G4_telo_D } \\
\text { NA_6xSG }\end{array}$ \\
\hline s.e.m. & 0.016 & 0.093 & 0.19 & 0.24 & 0.29 & 0.20 & 0.16 & 0.11 & 0.10 & 0.24 & 0.086 & 0.30 & 0.31 & 0.37 \\
\hline
\end{tabular}


8 Supplementary Table 3 | Statistics for RT-qPCR data (48 hr time point), from Fig. 2c and Supplementary Fig. 5b.

\begin{tabular}{|c|c|c|c|c|c|c|c|c|c|c|c|c|c|c|c|c|c|c|c|}
\hline $\begin{array}{l}\text { GFP/(mCherry } \\
\text { +hActb), } \\
\text { normalized to } \\
\text { "Mock ligation" }\end{array}$ & $\begin{array}{l}\text { Mock } \\
\text { ligation }\end{array}$ & $\begin{array}{l}\text { No } \\
\text { ligati } \\
\text { on }\end{array}$ & $\begin{array}{l}\text { 29rA } \\
\text { _ddC }\end{array}$ & $\begin{array}{c}\text { 3xSrA } \\
\text { dddC }\end{array}$ & $\begin{array}{l}\text { 3xSrA_- } \\
\text { InvdT }\end{array}$ & $\begin{array}{l}\text { 3xSrG_- } \\
\text { InvdT }\end{array}$ & $\begin{array}{l}\text { 6xSr( } \\
\text { AG) }\end{array}$ & $\begin{array}{r}\text { 3xdA } \\
\text { ddCC }\end{array}$ & $\begin{array}{l}23 x d A \\
\text { _ddC }\end{array}$ & $\begin{array}{l}\text { G4_t } \\
\text { elo_D } \\
\text { NA_ } \\
\text { GtoC }\end{array}$ & $\begin{array}{l}\text { G4_t } \\
\text { elo_D } \\
\text { NA_- } \\
\text { WT }\end{array}$ & $\begin{array}{l}\text { G4_C9 } \\
\text { orf72_- } \\
\text { RNA_6 } \\
\text { xSrG }\end{array}$ & $\begin{array}{l}\text { G4_C9 } \\
\text { orf72_- } \\
\text { DNA_- } \\
\text { 6xSG }\end{array}$ & $\begin{array}{l}\text { G4_telo } \\
\text { DNA_- } \\
\text { 6xSG }\end{array}$ & $\begin{array}{l}\text { mC } \\
\text { herr } \\
y \\
\text { only }\end{array}$ & & & & \\
\hline Mean & 1.09 & 0.92 & 0.76 & 1.67 & 1.15 & 1.51 & 2.13 & 0.82 & 0.91 & 1.11 & 0.99 & 2.37 & 2.39 & 2.38 & $\begin{array}{r}0.000 \\
5\end{array}$ & & & & \\
\hline s.d. & 0.50 & 0.28 & 0.13 & 0.11 & 0.24 & 0.20 & 0.29 & 0.05 & 0.04 & 0.25 & 0.08 & 0.57 & 0.60 & 0.11 & $\begin{array}{r}0.000 \\
1\end{array}$ & & & & \\
\hline $\begin{array}{l}\text { IfnB1 / hActb, } \\
\text { normalized to } \\
\text { "Mock ligation" }\end{array}$ & $\begin{array}{l}\text { Mock } \\
\text { ligation }\end{array}$ & $\begin{array}{l}\text { No } \\
\text { ligati } \\
\text { on }\end{array}$ & $\begin{array}{r}29 r A \\
\text { ddC }\end{array}$ & $\begin{array}{l}\text { 3xSrA } \\
\text { _ddC }\end{array}$ & $\begin{array}{l}\text { 3xSrA_- } \\
\text { InvdT }\end{array}$ & $\begin{array}{l}\text { 3xSrG_- } \\
\text { InvdT }\end{array}$ & $\begin{array}{l}\text { 6xSr( } \\
\text { AG) }\end{array}$ & $\begin{array}{r}3 x d A \\
\text { dddC }\end{array}$ & $\begin{array}{l}23 x d A \\
\text { _ddC }\end{array}$ & $\begin{array}{l}\text { G4_t } \\
\text { elo_D } \\
\text { NA_ } \\
\text { GtoC }\end{array}$ & $\begin{array}{l}\text { G4_t } \\
\text { elo_D } \\
\text { NA_- } \\
\text { WT }\end{array}$ & $\begin{array}{l}\text { G4_C9 } \\
\text { orf72_- } \\
\text { RNA_6 } \\
\text { xSrG }\end{array}$ & $\begin{array}{l}\text { G4_C9 } \\
\text { orf72_- } \\
\text { DNA_- } \\
\text { 6xSG }\end{array}$ & $\begin{array}{l}\text { G4_telo } \\
\text { DNAA_- } \\
\text { 6xSG }\end{array}$ & $\begin{array}{l}\mathrm{mCh} \\
\text { erry } \\
\text { only }\end{array}$ & $\begin{array}{l}\text { Unmo } \\
\text { dified } \\
\text { GFP } \\
\text { mRN } \\
\text { A }\end{array}$ & \begin{tabular}{|l|}
200 \\
ng \\
poly ( \\
I:C)
\end{tabular} & $\begin{array}{l}500 \\
\text { ng } \\
\text { poly }( \\
\text { I:C) }\end{array}$ & $\begin{array}{l}\text { Transf } \\
\text { ection } \\
\text { only }\end{array}$ \\
\hline Mean & 1.02 & 1.42 & 1.20 & 1.09 & 1.15 & 1.24 & 1.23 & 1.15 & 1.28 & 1.25 & 1.74 & 1.28 & 1.10 & 1.31 & 1.04 & 3.24 & 40 & 100 & 2 \\
\hline s.d. & 0.20 & 0.73 & 0.37 & 0.41 & 0.38 & 0.28 & 0.41 & 0.45 & 0.58 & 0.27 & 0.86 & 0.45 & 0.44 & 0.40 & 0.27 & 0.37 & 6 & 20 & 1 \\
\hline
\end{tabular}

9 
10 Supplementary Table 4 | Statistics for GFP/mCherry fluorescence ratio data, from Fig. 5a (normalized to "mock ligation" samples).

\begin{tabular}{|l|l|l|l|}
\hline 24 hour statistics & mCherry only & Untreated mRNA & 6xSr(AG) \\
\hline Mean & 0.24 & 1.00 & 10.15 \\
\hline s.d. & 0.19 & 0.19 & 1.90 \\
\hline s.e.m. & 0.04 & 0.04 & 0.45 \\
\hline Sample Size & 12 & 12 & 12 \\
\hline 48 hr statistics & mCherry only & Untreated mRNA & 6xSr(AG) \\
\hline Mean & 0.47 & 1.00 & 10.61 \\
\hline s.d. & 0.32 & 0.26 & 2.10 \\
\hline s.e.m. & 0.07 & 0.06 & 0.49 \\
\hline Sample Size & 12 & 12 & 12 \\
\hline
\end{tabular}

